\title{
T-cell infiltrates and tumor budding: promising prognostic factors in the tumor microenvironment of colorectal cancer
}

\author{
Koelzer, Viktor H ; Lugli, Alessandro
}

\begin{abstract}
The tumor microenvironment is the battleground of pro- and anti-tumor factors. On the tumor's side, epithelial-mesenchymal transition (EMT) and tumor budding is the histomorphologic signature of aggressive disease. On the host's side, T-effector cell infiltrates are the hallmark of an active anti-tumoral immune defense. These morphological features can be measured by the histopathologist and represent important novel histomorphological prognostic factors in colorectal cancer (CRC).
\end{abstract}

DOI: https://doi.org/10.2217/crc.13.26

Posted at the Zurich Open Repository and Archive, University of Zurich

ZORA URL: https://doi.org/10.5167/uzh-174584

Journal Article

Published Version

Originally published at:

Koelzer, Viktor H; Lugli, Alessandro (2013). T-cell infiltrates and tumor budding: promising prognostic factors in the tumor microenvironment of colorectal cancer. Colorectal Cancer, 2(3):173-176.

DOI: https://doi.org/10.2217/crc.13.26 


\section{T-cell infiltrates and tumor budding: promising prognostic factors in the tumor microenvironment of colorectal cancer}

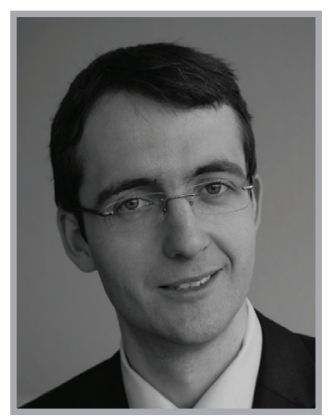

Viktor H Koelzer ${ }^{1}$

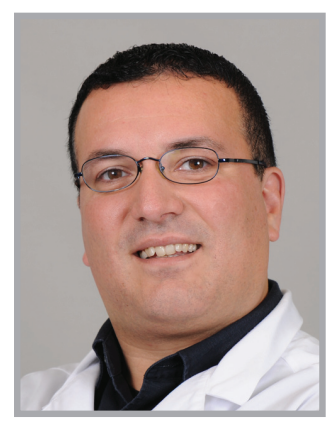

Alessandro Lugli*1
"The balance of pro- and anti-tumor factors may ... define whether local tumor progression will result in breach of the vascularity and systemic spread of disease or whether the neoplastic cell population can be contained by an active anti-tumoral effector and memory T-cell response."
The tumor microenvironment is the battleground of pro- and anti-tumor factors. On the tumor's side, epithelial-mesenchymal transition (EMT) and tumor budding is the histomorphologic signature of aggressive disease. On the host's side, T-effector cell infiltrates are the hallmark of an active anti-tumoral immune defense. These morphological features can be measured by the histopathologist and represent important novel histomorphological prognostic factors in colorectal cancer (CRC).

\section{The tumor microenvironment in colorectal cancer}

On the battleground, the picket lines correlate to the resources available to each warring party that are strategically employed in the terrain at stake. In the tumor microenvironment, a constantly growing mass of malignant cells infiltrates the locoregional tissue, reaching the opportunity for systemic spread when lymphovascular spaces are breached. Recent studies highlight that epithelial tumors undergo mesenchymal transition in the tumor microenvironment, allowing dissociative growth, migration and lysis of stromal components [1]. It is likely that single tumor cells in the tumor microenvironment permeate vessels and may be the seed for distant metastatic disease, which is the eventual cause of death for many cancer patients [2]. This hypothesis is supported by data demonstrating stem cell-like features in cells undergoing EMT [3], and evidence of a reverse process of mesenchymal-epithelial transition in sites of metastasis [2]. In CRC, the visible snapshot of cells undergoing EMT is the presence of single cells or small clusters of up to five cells in the tumor stroma ahead of the invasive front, termed tumor buds [4].

Infiltrating tumor buds and the main tumor are subject to constant immunoselective pressure from the adaptive antitumor immunity, which recognizes aberrant antigen profiles, and is capable of mounting an efficient antitumoral immune response. Strong experimental and translational evidence suggests that efficient immune

\footnotetext{
"Recent studies highlight that epithelial tumors undergo mesenchymal transition in the tumor microenvironment, allowing dissociative growth, migration and lysis of stromal components."
}

'Clinical Pathology Division \& Translational Research Unit, Institute of Pathology, University of Bern, Murtenstrasse 31, $\mathrm{CH}-3010$ Bern, Switzerland

*Author for correspondence: Tel.: +41 31632 99 58; Fax: +41 31632 49 95; alessandro.lugli@pathology.unibe.ch 
“...definite consensus on the optimal method for assessment has to be reached urgently to carry this powerful prognostic factor into practice" rejection of a tumor shows a characteristic, replicable profile with highly conserved patterns between different patients and even different tumor types [5]. Recently, this host-related factor has been made accessible for histopathologic quantification through the development of the 'immunoscore' [6]. The use of the immunoscore in CRC has demonstrated two central findings [7]. First, patients with a strong in situ immune reaction to CRC have infrequent metastatic spread of disease and a significantly improved disease-free and overall survival. Second, the prognostic value of the immunoscore may offer superior discriminatory power to TNM staging and is independent of the anatomic extent of the tumor.

\section{Budding: the tumor-related factor}

Tumor budding was first described by Jeremy Jass in 1986, and has been well characterized as a reproducible and frequent feature of CRC with a highly aggressive clinical course [8]. The presence of tumor buds in CRC is an independent predictive factor of local recurrence of disease, lymphovascular invasion, nodal and distant metastasis, and poor disease-specific survival [9]. This independent predictive information may be of decisive value in central clinical settings. First, the presence of tumor budding in endoscopically removed malignant polyps may be an indicator of locoregional spread of disease requiring segmental resection [10]. Second, the presence of tumor budding may identify patients who follow an aggressive clinical course in locally advanced, node-negative, stage 2 disease who may profit from chemotherapy to prevent distant disease recurrence [11]. Third, tumor budding may represent a novel histomorphological prognostic factor for application in biopsy samples, allowing effective risk stratification of CRC patients at the time of histopathological confirmation of diagnosis [12]. Consequently, the value of tumor buds in the tumor microenvironment has been recognized as an additional prognostic factor by WHO and the Union for International Cancer Control [13].

Translational research suggests that tumor budding at the invasive front of CRC is often found in tumors with activation of EMT-related signaling pathways; an increased expression of TGF- $\beta$ or loss of cell adhesins such as E-cadherin or syndecan-1 frequently accompanies the presence of tumor buds at the invasive front [9]. Furthermore, the presence of tumor buds is often detected in tumors with an increased capability for stromal degradation, characterized by increased expression of metalloproteinases, cathepsin B or urokinase receptor [9]. Tumor buds themselves frequently exhibit 'pseudopodia-like' cytoplasmic protrusions, which may be formed during the process of migration [14]. Interestingly, tumor buds also overexpress proteins related to stromal degradation as further evidence of their migratory potential [15]. Putative stem cell features have been linked to the protein expression of ABCG5 and EPCAM in tumor buds, which may allow the seeding of distant sites once the tumor vasculature is permeated [16].

The fact that tumor budding can be quantitated, shows powerful independent prognostic value and is related to aggressive disease biology suggests that scoring tumor buds should become part of the standard pathological assessment of CRC. Much like the Gleason score for prostate cancer, or the Bloom-Richardson-Elston score for breast cancer, the prognostic power of tumor budding could therefore be harnessed for daily diagnostic practice in gastrointestinal pathology. Central to the establishment of a novel predictive factor is the ease of application and interobserver reproducibility; substantial evidence suggests that scoring tumor buds in a clearly defined area is superior to the qualitative, subjective assessment of tumor budding 'intensity' in terms of reproducibility and predictive value for adverse outcome [17]. Furthermore, tumor buds can be assessed in conventional hematoxylin and eosinstained slides but scoring can be facilitated by using pan-cytokeratin immunohistochemistry [17]. As additional prognostic markers are highly valuable for therapy stratification, definite consensus on the optimal method for assessment has to be reached urgently to carry this powerful prognostic factor into practice.

\section{T-cell infiltrates: the host-related factor}

According to the TNM classification of CRC specimens, we currently focus on the anatomic extension of the tumor itself for prognostication [13]. Even though well proven, this approach does not take any characteristics of the host response to the cancer into consideration. In particular, no measure of the antitumoral immune response is provided in standard histopathologic assessment of CRC. Yet, strong evidence suggests that the activity of the antitumoral immune defense plays a major role for survival outcome of CRC patients [6,7]. However, no immunebased prognostic factors have been established 
in clinical practice since the first publication on intratumoral immune infiltrates in CRC by Jass in 1986 [18]. In fact, we may have spent 25 years of looking preferentially at one side of the histopathologic picture of CRC.

This may soon change. It is now recognized that the presence of intra- and peri-tumoral $\mathrm{CD}^{+}$cytotoxic and $\mathrm{CD} 45 \mathrm{RO}^{+}$memory $\mathrm{T}$ lymphocytes are characteristic of an inflamed tumor microenvironment and active immune rejection by the host. A new, possibly transformational approach has been developed in CRC research that emphasizes the role of the tumor-host interaction and gauges the activity of the antitumoral immune defense [6]. The so-called 'immunoscore' allows histologic quantification of tumor infiltrating immune cells in the tumor center and the tumor margins [6,7]. A substantial number of retrospective clinical trials were published over the last decade demonstrating that in situ analysis of immune cell infiltrates in any region of CRC carries independent prognostic value for the patient. In fact, immune cells within tumor samples were found to be a better predictor of patient survival than the TNM system [7]. This may seem surprising, but it should be noted that an increased frequency and activation of effector $T$ cells in the tumor microenvironment is linked to the complex process of an active antitumoral defense. In these terms, an inflamed tumor microenvironment is characterized by STAT-1/IRF1/IRF5 activity, inflammatory Th1based cytokine expression and presence of T-cell attractants (CXCR3 and CCR5 ligands) [5]. This intricate modulation of the tumor microenvironment converges in the presence of intratumoral and stromal effector $\mathrm{T}$ cells, which can then be quantified by the histopathologist.

As with tumor budding, the ease of assessment and reproducibility of the immunoscore will play a central role for use in daily diagnostics. In an elegant approach, standardized

\section{References}

1 De Craene B, Berx G. Regulatory networks defining EMT during cancer initiation and progression. Nat. Rev. Cancer 13(2), 97-110 (2013).

2 Gao D, Vahdat LT, Wong S, Chang JC, Mittal V. Microenvironmental regulation of epithelial-mesenchymal transitions in cancer. Cancer Res. 72(19), 4883-4889 (2012).

3 Scheel C, Weinberg RA. Cancer stem cells and epithelial-mesenchymal transition:

software applications have been developed to allow objective and reproducible assessment of immune cell infiltrates [7]. Furthermore, international collaborative efforts are currently under way to validate the immunoscore in CRC in a large multinational, retrospective study [6].

\section{Future perspective}

The tumor microenvironment holds two promising prognostic factors that may allow detailed assessment of the balance of tumor growth and host defense. Based on continuous efforts of CRC researchers, an integration of both factors into daily diagnostic practice may seem plausible within the near future. The balance of pro- and anti-tumor factors may therefore define whether local tumor progression will result in breach of the vascularity and systemic spread of disease or whether the neoplastic cell population can be contained by an active anti-tumoral effector and memory T-cell response. Furthermore, combined assessment of the histomorphological features of the tumor-host interface may allow visual integration of the biological aggressiveness of disease and the strength of the antitumoral immune defense in an 'attacker-defender' approach [19]. Consequently, prognostication based on both tumor budding as a tumorrelated factor and the immune infiltrates as a host-related factor could represent a promising translational approach for future studies.

\section{Financial \& competing interests disclosure}

The authors have no relevant affiliations or financial involvement with any organization or entity with a financial interest in or financial conflict with the subject matter or materials discussed in the manuscript. This includes employment, consultancies, honoraria, stock ownership or options, expert testimony, grants or patents received or pending, or royalties.

No writing assistance was utilized in the production of this manuscript.

concepts and molecular links. Semin. Cancer Biol. 22(5-6), 396-403 (2012).

4 Prall F. Tumour budding in colorectal carcinoma. Histopathology 50(1), 151-162 (2007).

5 Immunologic Signatures of Rejection. Marincola FM, Wang E (Eds). Springer, NY, USA (2011).

6 Galon J, Pages F, Marincola FM et al. The immune score as a new possible approach for the classification of cancer. J. Trans. Med. 10, 1 (2012).

\section{“...prognostication based on both tumor budding \\ as a tumor-related factor and the immune infiltrates as a \\ host-related factor could represent a promising translational approach for future studies."}

7 Mlecnik B, Tosolini M, Kirilovsky A et al. Histopathologic-based prognostic factors of colorectal cancers are associated with the state of the local immune reaction. J. Clin. Oncol. 29(6), 610-618 (2011).

8 Jass JR, Atkin WS, Cuzick J et al. The grading of rectal cancer: historical perspectives and a multivariate analysis of 447 cases. Histopathology 10(5), 437-459 (1986).

9 Zlobec I, Lugli A. Epithelial mesenchymal transition and tumor budding in aggressive colorectal cancer: tumor budding as 
EDITORIAL Koelzer \& Lugli

oncotarget. Oncotarget 1(7), 651-661

(2010).

10 Ueno H, Mochizuki H, Hashiguchi Y et al. Risk factors for an adverse outcome in early invasive colorectal carcinoma.

Gastroenterology 127(2), 385-394 (2004).

11 Wang LM, Kevans D, Mulcahy $\mathrm{H}$ et al. Tumor budding is a strong and reproducible prognostic marker in T3N0 colorectal cancer. Am. J. Surg. Pathol. 33(1), 134-141 (2009).

12 Giger OT, Comtesse SC, Lugli A, Zlobec I, Kurrer MO. Intra-tumoral budding in preoperative biopsy specimens predicts lymph node and distant metastasis in patients with colorectal cancer. Modern Pathol. 25(7), 1048-1053 (2012).
13 Bosman FT. WHO Classification of Tumours of the Digestive System (4th Edition).

International Agency for Research on Cancer, Lyon, France (2010).

14 Shinto E, Jass JR, Tsuda H et al. Differential prognostic significance of morphologic invasive markers in colorectal cancer: tumor budding and cytoplasmic podia. Dis. Colon Rectum 49(9), 1422-1430 (2006).

15 Guzinska-Ustymowicz K. MMP-9 and cathepsin B expression in tumor budding as an indicator of a more aggressive phenotype of colorectal cancer (CRC). Anticancer Res. 26(2B), 1589-1594 (2006).

16 Hostettler L, Zlobec I, Terracciano L, Lugli A. ABCG5-positivity in tumor buds is an indicator of poor prognosis in node-negative colorectal cancer patients. World J. Gastroenterol. 16(6), 732-739 (2010).

17 Horcic M, Koelzer VH, Karamitopoulou E et al. Tumor budding score based on 10 high-power fields is a promising basis for a standardized prognostic scoring system in stage II colorectal cancer. Hum. Pathol. 44(5), 697-705 (2012).

18 Jass JR. Lymphocytic infiltration and survival in rectal cancer. J. Clin. Pathol. 39(6), 585-589 (1986).

19 Lugli A, Karamitopoulou E, Panayiotides I et al. CD8+ lymphocytes/tumour-budding index: an independent prognostic factor representing a 'pro-/anti-tumour' approach to tumour host interaction in colorectal cancer. Br. J. Cancer 101(8), 1382-1392 (2009). 